Progress in Oceanography

September 2018, Volume 166, Pages 2-3

http://dx.doi.org/10.1016/i.pocean.2018.03.015

http://archimer.ifremer.fr/doc/00434/54607/

(c) 2018 Elsevier Ltd. All rights reserved.

\title{
Multidisciplinary integrated surveys: objectives, achievements and future directions
}

\author{
Petitgas Pierre ${ }^{1,{ }^{*}}$, Santos Vásquez Begoña ${ }^{2}$ \\ ${ }^{1}$ IFREMER, Centre Atlantique, Research Unit EMH, rue de l'île d'Yeu, 44300 Nantes, France. \\ 2 IEO, Centro de Vigo, Subida a Radio Faro 50, 36390 Vigo, Spain. \\ * Corresponding author : Pierre Petitgas, email address : pierre.petitgas@ifremer.fr
}

\section{Highlights}

- Integrated surveys developed by adding new sampling protocols to existing surveys. They are a response to integrated fisheries and environment policies. They provide relevant data for ecosystem description and assessment. Next steps will concern integration, automation, and big-data analytical framework. 


\section{Preface}

Multidisciplinary integrated surveys: objectives, achievements and future directions

\section{Introduction}

Over the last two decades, fisheries surveys have shifted their focus, from providers of data for single fish stock assessments to become platforms for the multidisciplinary integrated monitoring of ecosystems (including the continued provision of the data necessary for assessing fish stocks). Such change in focus was possible in part because new research vessels built in the 1990s were sufficiently large and equipped for multidisciplinary teams to work simultaneously onboard. The shift in survey objectives, and the data provided, supported changes in policies for implementing ecosystem based approaches. In many countries around the Atlantic, sectoral fisheries management is now embedded into ecosystem conservation and environmental legislation. Similarly, integrated maritime policies now require collecting data on major ecosystem components to monitor the achievement of good environmental status. Presently, fisheries surveys undertaken yearly over large marine areas have become the multidisciplinary platforms providing the required data.

\section{Overview of themes in this issue}

This Special Issue (SI) highlights solutions found for implementing multidisciplinary integrated surveys, as well as major results obtained.

\section{Surveys}

Two papers, in the Barents Sea (Eriksen et al., this issue) and the Bay of Biscay (Doray et al. (a), this issue), detail the historical and technical developments that allowed this process. The other papers in the SI present key results stemming from the multidisciplinary spring pelagic surveys undertaken in the Bay of Biscay since 2000 by Spain and France. The surveys are part of the national monitoring programmes, which are national contributions to two European Union policy frameworks, the (sectoral) Common Fisheries Data Collection and the (environmental) Marine Strategy Framework Directive. The surveys are coordinated under the auspices of the International Council for the Exploration of the Sea (ICES), where scientists standardize survey data collection, data analysis methodologies, and deliver data products for assessing fish stocks and ecosystems.

\section{Instruments and Analytical Methods}

Several papers in this SI describe specific methods for integrated monitoring and data analysis. The development of multidisciplinary surveys has required the integration and optimization of various sampling protocols at different scales. In the Barents Sea, changes in survey design and sampling strategy resulted in ecosystem monitoring at large scales, with particular emphasis given to selected fish stocks (Eriksen et al., this issue). An example of optimization in the collection of data is the Zoocam (Colas et al., this issue). This imaging system technology provides en route continuous and automated high resolution spatial count and morphometric data of plankton groups. With this technology, a new stream of survey data is created. The multidisciplinary integrated surveys can now be envisioned as part of regional integrated monitoring strategies, where buoys, drones, satellites and surveys collect in an automated way information at complementary spatio-temporal scales. In that context, Perrot et al. (this issue) describe how surveys have been used to provide the groundtruthing data necessary to interpret satellite images.

Phenology and variability of the ecosystem components 
An important issue for yearly repeated monitoring surveys is the difference between calendar and ecological schedules. Surveys taking place on the same dates each year may not always coincide with the timing of ecological processes. Huret et al. (this issue) suggest ways to quantify these variations and how to best interpret the resulting inter-annual differences. Also, it is important to keep in mind that multidisciplinary integrated surveys collect data from trophic levels with different dynamics. This was highlighted by Petitgas et al. (this issue) who showed how the gradients in lower trophic levels in the Bay of Biscay - at the scale of the survey area - were strong enough to characterize timeconsistent spatial patterns across all trophic levels, thus allowing for mapping ecosystem spatial structure as a whole. Other papers dealt with specific aspects of the methodology, i.e. the spatiotemporal and multivariate data analysis methods needed to characterize changes in the different ecosystem components (Petitgas et al., this issue; Doray et al. (b), this issue) or of the data, i.e., marine mammal observations taken onboard the surveys were validated as being useful to assess trends in abundance time series (Saavedra et al., this issue). In addition to maps, survey products include time series of many indices of the different ecosystem components. Multiple trend analysis was applied to times series of megafauna abundance (Authier et al., this issue). Another approach was to rank time series' indices based on their autocorrelation and identify those indices that drove ecosystem change as a whole (Doray et al. (c), this issue).

\section{Ecosystem function and energy budgets}

Other papers in this SI provide knowledge of the functioning of the ecosystem in the Bay of Biscay and highlight how new data, in addition to standard fisheries data, can provide information about trophic processes, characterize the habitats of pelagic fishes and top predators, as well as assess their trends in abundance. Energy fluxes across trophic levels were analyzed by means of energy density, a valuable measure that can be easily collected during surveys. Measurements of energy density in zooplankton organisms (Dessier et al., this issue) revealed important differences between taxonomic groups, which could influence the energy transfer to pelagic fish. Condition in sardine and anchovy determined with energy density measurements (Gatti et al., this issue) revealed differences in feeding regimes between the two species, with sardine showing a greater energy storage capacity. Also, energy storage in sardine differed between habitats. The trophic position of sardine as characterized with stable isotopes (Bode et al., this issue) was reported to depend also on population level abundance, meaning that feeding regimes and resource availability were dynamic. The consistency and variability in time of habitats of top predators (Lambert et al., this issue) and of pelagic fish (Doray et al., this issue) were documented and related to environmental parameters.

\section{Next steps for integrated surveys}

Multidisciplinary integrated surveys have developed by adding new objectives and sampling protocols to existing fishery surveys, each relevant for a particular ecosystem component. This approach has been transformational. Abundance indices for specific fish stocks are now embedded in a larger ecosystem context with survey data contributing to characterize ecosystem structure and patterns of variability. More process understanding and less structural description is now needed, which will require a coherent monitoring strategy across ecosystem components and the development of an analytical framework. We foresee three major next steps in the continued development of integrated surveys, which will unlock sampling at high resolution, and enhance our understanding of the relationships across ecosystem components. We anticipate the future steps will develop around the key concepts of integration, automation, and big data analytical framework

The integration of observational scales. The domain-wide integration of pelagic and demersal surveys performed in different seasons, with similar protocols for the lower trophic levels, will allow joint 
analyses of seasonal and interannual variability, as well as of the bentho-pelagic coupling. Such integrated strategies over the entire annual cycle will allow better characterization of habitats with a full life-cycle approach, and thus will also allow the evaluation of climate change impacts. In addition, the integration of survey data with data collected year-round at fixed observatories or with drones should allow the analysis of intra-seasonal variability at finer scales.

The automated acquisition of novel data. The use of high resolution data (satellite, in-situ imaging, broadband acoustics) will unveil fine-scale plankton and fish distribution and abundance patterns over large areas and enable to better understand the relationship between plankton production and fish condition. Also, new protocols already exist for the automated acquisition of novel fish data: functional traits can be measured with 3D scanners, energy density with probes, genetic methods can identify prey species from stomach contents, etc. This will make possible a coherent monitoring of corresponding features across trophic levels.

An integrated analytical framework. The analysis of such datasets at individual and community levels will require new capabilities for management of big data flows and efficient analytical algorithms, e.g., for deep learning. These novel data will enhance our understanding of trophic relationships and trade-offs in habitats occupied, in space and time.

In all, we hope this SI will be a milestone in the development of multidisciplinary ecosystem surveys. The examples of the data products generated and the knowledge gained from these multidisciplinary surveys highlight that, in addition to the provision of abundance indices for fish stock assessments, they are invaluable platforms for the characterization, assessment, and monitoring of the marine ecosystems.

\section{Acknowledgements}

The coordination of the Spanish and French integrated pelagic surveys in the Bay of Biscay started in 2000 with the support of the EU project Pelasses (DG-Mare project no. 99/010), which also involved Portugal. This $\mathrm{SI}$ is in part the result of this adventure, still ongoing. ICES (International Council for the Exploration of the Sea) has been instrumental in this endeavour by providing a forum for the teams involved in the surveys to meet annually. We would like to acknowledge the authors who contributed to the SI on a diversity of topics, thus providing a comprehensive overview. We would also like to thank the Editor-in-Chief, Dr. Francisco Werner, for his support when designing the $\mathrm{SI}$, and his patience and suggestions during the edition of the papers that form part of this SI.

\section{References}

Authier, M. et al. This issue. Exploring change in the relative abundance of marine megafauna in the Bay of Biscay, 2004-2016.

Bode, A. et al. This issue. A trophic index for sardine (Sardina pilchardus) and its relationship to population abundance in the southern Bay of Biscay and adjacent waters of the NE Atlantic

Colas, F. et al. This issue. The ZooCAM, a new in-flow imaging system for fast onboard counting, sizing and classification of fish eggs and metazooplankton

Dessier, A. et al. Variability of energy density among mesozooplankton community: New insights in functional diversity to forage fish. 
Doray, M. et al. (a). This issue. The PELGAS survey: Ship-based integrated monitoring of the Bay of Biscay pelagic ecosystem.

Doray, M. et al. (b). This issue. Spring habitats of small pelagic fish communities in the Bay of Biscay.

Doray, M. et al. (c). This issue. Monitoring small pelagic fish in the Bay of Biscay ecosystem, using indicators from an integrated survey.

Eriksen, E. et al. This issue. From single species surveys towards monitoring of the Barents Sea ecosystem.

Gatti, P. et al. This issue. Bioenergetic condition of anchovy and sardine in the Bay of Biscay and English Channel.

Huret, M. et al. This issue. Survey timing vs. ecosystem scheduling: Degree-days to underpin observed interannual variability in marine ecosystems.

Perrot, L. et al. This issue. Coccolith-derived turbidity and hydrological conditions in May in the Bay of Biscay.

Petitgas, P. et al. This issue. Ecosystem spatial structure revealed by integrated survey data.

Saavedra, C. et al. This issue. Assessing the environmental status of the short-beaked common dolphin (Delphinus delphis) in North-western Spanish waters using abundance trends and safe removal limits.

Spitz, J. et al. This issue. Prey consumption by cetaceans reveals the importance of energy-rich food webs in the Bay of Biscay.

Pierre Petitgas *

IFREMER, Centre Atlantique, Research Unit EMH, rue de l'île d'Yeu, 44300 Nantes, France.

*Corresponding author. Tel: +33240374163. Fax:+33240374170. E-mail: pierre.petitgas@ifremer.fr

Begoña Santos Vásquez

IEO, Centro de Vigo, Subida a Radio Faro 50, 36390 Vigo, Spain. 
PROOCE_2018_49_R1

Preface to the SI on Integrated surveys

Highlights

Multidisciplinary integrated surveys developed by adding new sampling protocols to existing fishery surveys, each relevant for the major ecosystem components

They are a response to integrated fisheries and environment policies

They provide relevant data for ecosystem description and assessment

The next steps will develop around the concepts of integration, automation, and big data analytical framework 